Heme oxygenase-1 (HO-1, encoded by $H M O X 1)$ through degradation of pro-oxidant heme into carbon monoxide (CO), ferrous ions $\left(\mathrm{Fe}^{2+}\right)$ and biliverdin, exhibits cytoprotective, anti-apoptotic and anti-inflammatory properties. All of these potentially beneficial functions of HO-1 may play an important role in tumors' development and progression. Moreover, HO-1 is very often upregulated in tumors in comparison to healthy tissues, and its expression is further induced upon chemo-, radio- and photodynamic therapy, what results in decreased effectiveness of the treatment. Consequently, $\mathrm{HO}-1$ can be proposed as a therapeutic target for anticancer treatment in many types of tumors. Nonetheless, possibilities of specific inhibition of HO-1 are strongly limited. Metalloporphyrins are widely used in in vitro studies, however, they are unselective and may exert serious side effects including an increase in HMOX1 mRNA level. On the other hand, detailed information about pharmacokinetics and biodistribution of imidazole-dioxolane derivatives, other potential inhibitors, is lacking. The genetic inhibition of HO-1 by RNA interference (RNAi) or CRISPR/Cas9 approaches provides the possibility to specifically target HO-1; however, the potential therapeutic application of those methods are distant at best. In summary, HO-1 inhibition might be the valuable anticancer approach, however, the ideal strategy for HO-1 targeting requires further studies.

Key words: HO-1 inhibitors, siRNA, CRISPR/Cas9, anticancer strategy, tumorigenesis.

Contemp Oncol (Pozn) 2018; 22 (1A): 23-32 DOI: https://doi.org/10.5114/wo.2018.73879

\section{Heme oxygenase inhibition in cancers: possible tools and targets}

\author{
Paulina Podkalicka ${ }^{1 \star}$, Olga Mucha ${ }^{1 \star}$, Alicja Józkowicz ${ }^{1}$, Józef Dulak ${ }^{1,2}$, \\ Agnieszka Łoboda ${ }^{1,2}$
}

${ }^{1}$ Department of Medical Biotechnology, Faculty of Biochemistry, Biophysics and Biotechnology, Jagiellonian University, Krakow, Poland

${ }^{2}$ Malopolska Centre of Biotechnology, Jagiellonian University, Krakow, Poland

* Equal contributions.

\section{Biological functions of HO-1 - an overview}

Heme, an organic cofactor composed of iron and organic part called protoporphyrin IX, serves as a prosthetic group of many proteins. Hence, it facilitates a wide variety of processes like electron transport (cytochrome P450 family), enzyme catalysis (e.g. peroxidase, catalase, cyclooxygenase, nitric oxide synthase) and reversible binding of gases (hemoglobin, myoglobin, guanylate cyclase). Furthermore, it is involved in regulation of protein synthesis and cells differentiation [1]. From the other hand, free heme released from intracellular hemeproteins causes cellular and tissue damage. Toxic and pro-oxidant properties of heme are the consequences of iron-dependent reactive oxygen species (ROS) generation, lipids peroxidation and disruption of cellular membranes. As the result, an excessive level of circulating heme leads to several pathologies [2].

To overcome deleterious properties of heme, cellular machinery is equipped with heme oxygenases ( $\mathrm{HO})$, a microsomal enzymes which are responsible for the first, rate-limiting step in heme degradation pathway. The enzyme cleaves heme into three biological active products: carbon monoxide (CO), ferrous ions ( $\mathrm{Fe}^{2+}$ ) and biliverdin, which is subsequently converted into antioxidant bilirubin by biliverdin reductase. Molecular oxygen, cytochrome $\mathrm{P} 450$ reductase, and NADPH are required for the complete reaction [3]. So far, three isoforms of $\mathrm{HO}$ have been described - HO-1, HO-2 and $\mathrm{HO}-3$; however, the latter is rather the pseudogene originated from $\mathrm{HO}-2$ transcript and was identified only in rats [4]. Although both $\mathrm{HO}-1$ (encoded by HMOX1 gene) and HO-2 (encoded by HMOX2 gene) catalyze the same reaction in heme degradation pathway and share approximately $40 \%$ homology, they differ in various aspects, including the kinetics of catalyzed reaction and the level of their expression in selected tissues. Finally, what is the most important, $\mathrm{HO}-2$ is a constitutive isoform, whereas $\mathrm{HO}-1$ - inducible. According to that, $\mathrm{HO}-1$ is strongly upregulated in response to a wide spectrum of stimuli such as its substrate heme, proinflammatory cytokines, ROS, nitric oxide (NO), metalloporphyrins, heavy metals, prostaglandins, UV irradiation and many others. At the molecular level, expression of HO- 1 is mainly activated by the transcription factor Nrf2 (nuclear factor erythroid 2-related factor-2). Certain stimuli cause the release of Nrf2 from its inhibitor, Keap-1 (Kelch-like associating protein 1) and this lead to translocation of Nrf2 to the nucleus where it binds to antioxidant response element (ARE) located within the promoter of theHMOX1 gene. Moreover, several other transcription factors (e.g. AP-1, STAT and NF-אB) may be responsible for the induction of $\mathrm{HO}-1$ expression in stressful conditions, such as oxidative stress (reviewed in: [5, 6]).

Thus, except degradation of pro-oxidant heme, HO-1 exerts much broader and pleiotropic functions mediated by the end products of its activity. Carbon monoxide (CO) is a gasotransmitter which among several functions 
activates soluble guanylate cyclase (sGC) and cause vasodilation and inhibition of platelet aggregation. Through modulation of MAPK (mitogen-activated protein kinases) pathways, it exerts anti-inflammatory functions: decreases leukocyte adhesion, inhibits pro- and increases anti-inflammatory cytokines. Additionally, CO possess anti-proliferative and anti-apoptotic properties leading to protection

\section{Photodynamic therapy}

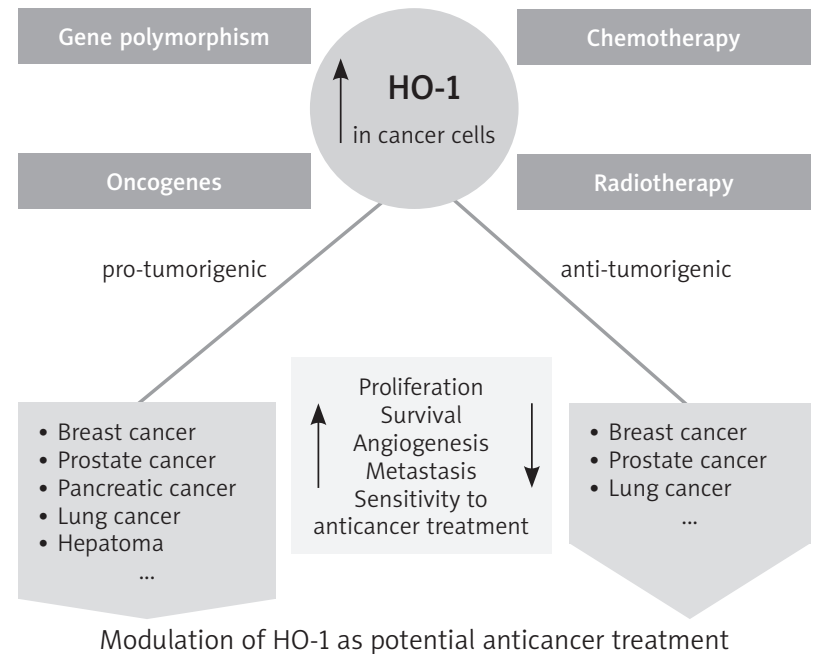

Fig. 1. HO-1 as a target for anticancer therapy. HO-1 expression in tumor tissues is increased as a consequence of oncogenic transformation, modified by HO-1 promoter polymorphism, and further upregulated in response to anticancer therapies. Generally, HO-1 exerts pro-tumorigenic effects through an increase in proliferation, survival, and angiogenesis. However, also the opposite, inhibitory effect on the progression of tumors have been reported in some tumor types

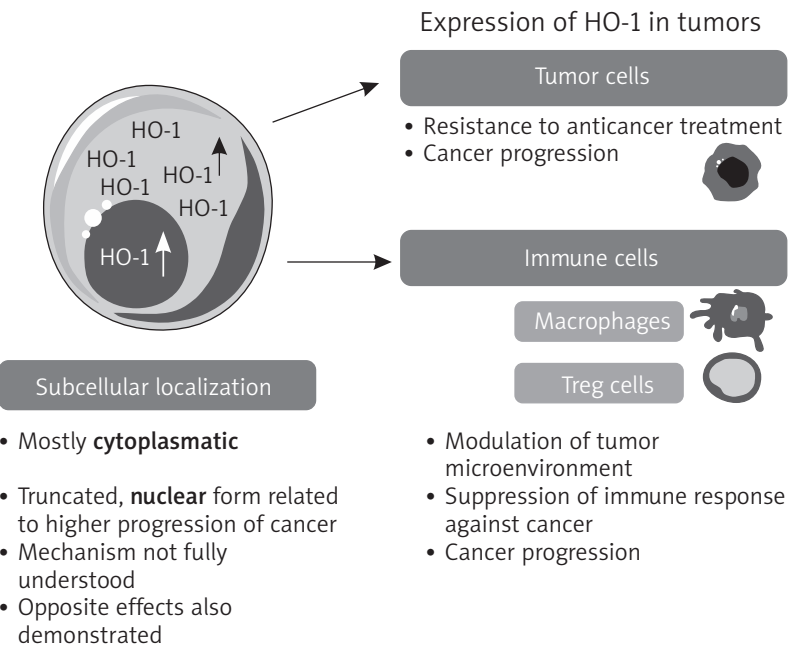

Fig. 2. Expression of HO-1 in tumors. Elevated level of HO-1 has been found in many types of tumors, both in cancer cells themselves and in immune cells such as macrophages and T regulatory cells, emphasizing its role in modulation of tumor microenvironment and evasion of the immune response against cancer. Interestingly, although HO-1 localizes mainly in cytoplasm, also its truncated, nuclear form has been linked with poor prognosis and progression of the disease in certain types of tumors; however, this mechanism is still not fully understood from tissue injury, however these functions are tissue-specific (reviewed in [6]). Moreover, its positive effect on angiogenic activity of endothelial and vascular smooth cells has been described [7, 8]. Ferrous ions increase the expression of ferritin, which is responsible for iron sequestration, hence it prevents toxicity caused by production of hydroxyl radicals. Additionally, $\mathrm{Fe}^{2+}$ ions induce iron transporter (Fe-ATPase) responsible for iron efflux. Finally, biliverdin and bilirubin possess anti-oxidant and anti-inflammatory properties by scavenging ROS, decreasing membrane lipids peroxidation, and inhibiting complement cascade (reviewed in: [6]).

\section{Emergent role of heme oxygenase-1 (HO-1) in tumor biology}

Unfortunately, all of the above mentioned cytoprotective actions of HO-1 and its by-products can be harmful, especially when translated into pathophysiological processes like tumorigenesis. Expression of HO-1 is significantly elevated in tumors, in comparison to healthy tissues, including pancreatic cancer, melanoma, renal cell carcinoma and others (reviewed in [9]). Noteworthy, expression of HO-1 can be further enhanced by anticancer therapies (including radio-, chemo- and photodynamic therapy) what often results in decreased effectiveness of the treatment [10-12] (Fig. 1).

It has to be stressed that the increased expression of HO-1 is found predominantly, but not only, in tumor cells (Fig. 2). Already in 1999, Nishie et al. have demonstrated the association of HO-1 with infiltrating macrophages and vascular densities in human gliomas [13]. HO-1 expression in the stromal macrophages has been also shown in the other cancer tissues. HO-1 expressed in FAP+/CD45+ inflammatory macrophages with a M2 phenotype was demonstrated to play a crucial role in immune suppression [14]. Moreover, HO-1-specific regulatory CD8+ T cells were detected both in peripheral blood of cancer patients and among tumor-infiltrating lymphocytes in situ of cancer patients [15]. HO-1-specific Treg cells were able to suppress immune response against cancer cells and efficiently inhibited T cell responses, including cytokine release, proliferation and cytotoxicity [15]. Interestingly, in melanoma, HO-1 was almost exclusively expressed in macrophages and the negligible expression was found in the tumor cells and other stromal cell types [16].

One important aspect of HO-1 effect on tumor biology might be related to its cellular localization. Generally, $\mathrm{HO}-1$ localizes mainly in the cytoplasm where it is associated with endoplasmic reticulum (ER), caveoli or mitochondria [17]; however, it was also found in the nucleus [18]. Such nuclear localization was observed in the lung [19], prostate [20] and head and neck cancer [21] and it correlated with poor prognosis and progression of the disease. Although the exact mechanism of such localization is not known yet, it was suggested that in response to stressful conditions, $\mathrm{HO}-1$ can be cleaved and translocated to the nucleus where it mediates upregulation of genes involved in anti-oxidant response and cytoprotection [18] (Fig. 2).

The exact role of HO-1 in tumor biology is multifaceted (Fig. 1). Although HO-1 is mostly shown to exert pro-tum- 
origenic properties by affecting cancer cells proliferation, survival and metastasis, also opposite effects have been described. Such anti-tumorigenic action of HO-1 favoring cancer development or/and survival was demonstrated in case of breast, prostate, pancreatic cancer and non-small cell lung carcinoma. This reflects complex and dual role of HO-1 in different types on tumors.

HO-1 can directly enhance proliferation of cancer cells what was shown in vitro and was further confirmed in vivo in the case of pancreatic cancer [10, 22], melanoma [23], and rhabdomyosarcoma [39]. Interestingly, Li et al. [24] have demonstrated that $\mathrm{HO}-1$ plays an important role in lung cancer carcinogenesis induced by tobacco-specific nitrosamine. An elevated level of $\mathrm{HO}-1$ was associated with increased proliferation of cancer cells through upregulation of p21, an inhibitor of apoptosis protein 2 (c-IAP2) and B-cell lymphoma-2 (Bcl-2) with a concomitant decrease in Bad protein. This, in turn, promoted the growth of cancer cells and prevented their death.

Also increased production of growth factors, such as epidermal growth factor (EGF) or elevated level of some mitogenic receptors like fibroblast growth factor receptor-1 (FGFR1) correlated with the accelerated proliferation of melanoma cancer cells [23]. However, also the opposite, anti-proliferative effect of $\mathrm{HO}-1$ was observed in breast cancer [25] and prostate cancer [26] where pharmacological inhibition of $\mathrm{HO}-1$ resulted in an augmented proliferation of cancer cells.

Cytoprotective and anti-apoptotic properties of $\mathrm{HO}-1$ in tumors are well known. In general, overexpression of HO-1 correlates with increased survival of cancer cells and their resistance to potentially toxic, anticancer drugs or therapies $[12,27]$. There are several possible mechanisms through which HO-1 may affect apoptosis of cancer cells. For instance, in gastric cancer, an elevated level of HO-1 contributed to decrease in caspase-3, the crucial mediator of apoptosis [28]. It was also demonstrated that HO-1 can directly inhibit apoptosis via activation of Akt (protein kinase B) pathway and this, in turn, can lead to increased ratio of antiapoptotic Bcl-2 (B-cell lymphoma-2) to proapoptotic Bax (Bcl-2-associated $X$ protein) proteins in colon cancer cell line [29]. Furthermore, genetic inhibition of HO-1 in breast cancer cell lines resulted in augmented doxorubicin-induced apoptosis via downregulation of antiapoptotic proteins [30]. However, some contradictory results showed that overexpression of HO-1 did not protect breast cancer cells from chemopreventive agents-induced apoptosis [31].

Interestingly, the role of autophagy in cancer biology is being widely studied and discussed nowadays, mostly due to its paradoxical role both in promoting and inhibiting cancer development and progression [32]. The link between $\mathrm{HO}-1$ and autophagy has been reported in renal cancer, as overexpression of $\mathrm{HO}-1$ induced by rapamycin and sorafenib treatment was reported to enhance survival of cancer cells by induction of anti-apoptotic B-cell lymphoma-extra large (Bcl-xL) protein and inhibition of autophagy [33]. On the other hand, upregulation of HO-1 in breast cancer cell lines exposed to doxorubicin treatment was accompanied with increased, cytoprotective autophagy via Scr-STAT3 signaling pathway [34].
HO-1 can directly support angiogenesis, the process defined as the formation of new blood vessels from preexisting vasculature, extremely important for tumor expansion and invasiveness. HO-1 can upregulate the expression or activity of proangiogenic factors like, among others, vascular endothelial growth factor (VEGF) (reviewed in: [35]) and stromal cell-derived factor-1 (SDF-1) [7]. Such proangiogenic effect of HO-1 was clearly demonstrated in glioma [13], pancreatic cancer [22], Kaposi sarcoma [36], melanoma [23], urothelial carcinoma [37], prostate cancer [38], and rhabdomyosarcoma [39]. Additionally, pharmacological inhibition of HO-1 resulted in suppressed angiogenesis and reduced VEGF level in mouse lung cancer in vivo [40].

Of importance, HO-1 overexpression may also contribute to enhanced metastasis as it was reported in the case of melanoma [23], pancreatic cancer [22] and squamous cell carcinoma [41]. Additionally, an elevated level of HO-1 in patients suffering from non-small cell lung cancer (NSCLC) was correlated with poor prognosis and enhanced metastasis occurrence [42]. However, in our hands, HO-1 overexpression in NSCLC NCl-H292 cells, caused not only decrease in their proliferation, migration and angiogenic potential, but also inhibition of tumors growth in vivo [43]. We observed that a high level of HO-1 in NSCLC cells correlated with a low expression of miR-378, the known oncomir and angiomir. Furthermore, treatment with CORM-2, CO-releasing molecule, decreased the expression of miR378 and consequently decreased expression of angiogenic mediators [43].

Another possible mechanism of HO-1 action might be connected with the effect on cell differentiation. Our initial experiments performed on murine $\mathrm{C} 2 \mathrm{C} 12$ myoblasts suggested that $\mathrm{HO}-1$ overexpression increases proliferation rate and the resistance to oxidative stress, however, it negatively regulates the differentiation of myoblasts to skeletal muscles [44]. Blockage of myotubes formation was independent of antioxidant properties of HO-1 but involved the inhibition of cEBPS-dependent expression of myoD and regulation of specific microRNAs, namely myomirs (miR-1, miR-133a, miR133b, and miR-206), responsible for myoblast differentiation. Downregulation of myomirs as well as other muscle regulatory factors including a master regulatory switch for myogenesis, MyoD and myogenin, by HO-1 overexpression was mimicked by CO-releasing molecule [44].

Based on such observations we performed experiments on rhabdomyosarcoma (RMS) tumor cell lines and primary RMS specimens. We observed the correlation of HO-1 expression with cancer invasiveness - much higher expression of HO-1 was detected in alveolar, more aggressive RMS (aRMS) than embryonal subtype. High expression of HO-1 was correlated with the decreased expression of miR-206, necessary for myoblasts differentiation [39]. In vivo, after subcutaneous injection of aRMA cell line and chemical inhibitor of $\mathrm{HO}$ activity application (SnPPIX), the growth of tumor was potently inhibited. What is more, the analysis of tumor vasculature indicated that $\mathrm{HO}-1$ inhibition exerted a potent anti-angiogenic effect. Lower number of CD-31 positive cells indicating decreased tumor vascularization was observed in SnPPIX-treated animals and those data were confirmed by in vivo ultrasonography im- 
aging. In accordance with those results, the comparison of primary RMS specimens showed that in $\mathrm{HO}-1$ overexpressing tumors were better vascularized. Further studies are necessary to fully describe the role of $\mathrm{HO}^{-1}$ in disturbed cell differentiation in other tumor types and to understand the molecular mechanism of the observed effects.

\section{Synthetic lethality of HMOX1 and FH genes}

A unique evidence suggesting the relevance of $\mathrm{HO}-1$ in tumor biology is its implication in hereditary leiomyomatosis and renal cell cancer (HLRCC) disease, a condition caused by inherited, autosomal dominant mutation in fumarate hydratase (FH) gene [45]. Mutation of FH, a tricarboxylic acid cycle enzyme, results in a permanently increased level of fumarate, which is proposed to act as an oncometabolite through a various mechanism [46].

Interestingly, Adam et al. demonstrated that HMOX1 expression was significantly upregulated both in $\mathrm{FH}$-deficient cells and in a mouse model of FH-deficiency [47]. Moreover, silencing of HMOX1 in FH-deficient cell lines resulted in their synthetic lethality [48]. This term refers to the situation in which simultaneous defect in two genes causes cell death, whereas at the same time separate dysfunction or mutation of each gene does not affect cells viability [49]. In silico study which was subsequently confirmed experimentally pointed out the importance of heme biosynthesis and degradation pathways in $\mathrm{FH}$-deficient cells linking glutamine uptake (as a source of carbon required for heme biosynthesis) with bilirubin excretion. Indeed, 18 out of 24 genes predicted as synthetically lethal with $\mathrm{FH}$, were associated with heme metabolism pathways. Importantly, genetic or pharmacological inhibition of HMOX1 significantly diminished survival of FH-deficient cells [48]. Also in our hands, genetic inhibition of HMOX1 in UOK262 cell line, which lacks FH activity, decreased cells viability, proliferation, and clonogenic potential (unpublished data). Hence, HMOX1 can serve as a potential therapeutic target also for patients suffering from HLRCC disease.

\section{Association between HO-1 gene polymorphism and cancer}

Noteworthy, genetic variation like polymorphism of HO-1 promoter and its association with cancer susceptibility was a subject of several studies. The human HMOX1 gene promoter in its $5^{\prime}$-flanking region contains a different number of GT microsatellite repeats, $(\mathrm{GT}) \mathrm{n}$, ranging from 11 to 40 [50]. Bimodal frequency distribution of (GT)n repeat lengths was observed in many populations studied, with the main alleles being 23 and 30 repeats (reviewed in: [6]). This genetic variability was shown to influence the response to exogenous stimuli, e.g. we showed that endothelial cells expressing the S allele (short, GT $\leq 23$ ) have high basal HO-1 expression and strong induction in response to hydrogen peroxide ( $\mathrm{H} 2 \mathrm{O} 2)$, lipopolysaccharide (LPS), prostaglandin J2(PGJ2) and cobalt protoporphyrin (CoPPIX) [51].

It has been demonstrated that shorter $(G T) n$ repeats within the HMOX1 promoter results in higher transcriptional activity and this, in turn, attributes to the increased risk and poor prognosis of pancreatic cancer [52] and gastric cancer [53]. Patients with a shorter version of the promotor are more susceptible to the development of pancreatic cancer and recurrence of the disease [52]. This data correlate with other results indicating higher $\mathrm{HO}-1$ expression in pancreatic tumors than in healthy tissue [10] and accelerated tumor growth, angiogenesis and metastatic potential of pancreatic cancer when $\mathrm{HO}-1$ is overexpressed [22].

On the other hand, the long (GT)n repeats in HMOX1 gene promoter was reported to correlate with a higher risk of breast cancer [54], esophageal squamous cell carcinoma [55] and laryngeal squamous cell carcinoma [56]. More complicated, other studies showed no correlation between HMOX1 polymorphism and the risk of lung squamous cell carcinoma [57] and sporadic colorectal cancer [58], indicating that the final outcome may depend on several factors including the type of the tumor, subject ethnicity, and many others. In accordance with all those various results, the meta-analysis of 10 studies involving 2,367 cases and 2,870 controls found no correlation between the length of (GT)n sequence and overall cancer risk although for some specific tumor types the association was reported [59].

\section{HO-1 as a therapeutic target in cancer - different ways of HO-1 inhibition}

As described above, inhibition of HO-1 may be considered as a mode for anticancer treatment. However, it cannot be rather effective as a single therapy, but most likely as an additional therapeutic strategy that could increase the sensitivity of the cancer cells to chemotherapy or radiotherapy. Inhibition of HO-1 can be achieved either through genetic tools or pharmacological agents (Fig. 3).

\section{Genetic inhibition of HO-1 expression (RNA interference, CRISPR/Cas9)}

RNA interference (RNAi) enables selective inhibition of desired genes on post-transcriptional, mRNA level. Small interfering RNA (siRNA) and short hairpin RNA (shRNA) are the most commonly used forms of RNAi whose action results in gene silencing either through cleavage of targeted mRNA, chromatin remodeling or blockage of protein synthesis. The usefulness of RNAi approach in studying gene functions has been widely reported in many areas of research including carcinogenesis (reviewed in: [60]).

Of importance, a substantial number of studies evaluating the role of HO-1 inhibition in cancer has been conducted using siRNA or shRNA sequences specifically targeting HO-1 transcript. Application of HO-1 siRNA in vitro resulted in beneficial increase in apoptosis of lung cancer cells [61], colon carcinoma cells [29], leukemic cells [62-64] and esophageal squamous carcinoma cells [65]. Furthermore, increased sensitivity to chemotherapeutics of pancreatic cancer cells [10], myeloid leukemia cells [66] and some other types of cancer cells was also reported (reviewed in: [27]). In contrast, a comprehensive study performed on prostate cancer cell line $\mathrm{PC} 3$ revealed rather pro-tumorigenic effect of HO-1 downregulation by siRNA, related to changes in cell-cell contact [67]. 
In vivo application of HO-1 siRNA/shRNA sequences or cancer cells modified by RNAi was also demonstrated. Direct, intraperitoneal injection of HO-1 siRNA resulted in the diminished growth of tumors in an orthotopic model of hepatocellular carcinoma [68]. Moreover, subcutaneous injection of human colon cancer cell line resistant to 5-fluorouracil (5-FU), transduced with shRNA against HO-1, significantly reduced tumor size and markedly increased the sensitivity of nude mice to 5-FU treatment [69].

Since its discovery, RNAi provided invaluable insight into molecular pathways and potential targets involved in tumor development and progression. Nonetheless, therapeutic application of RNAi remains limited by several factors related to variable efficiency and off-target effects, delivery of genetic material into specific tumor sites, questionable stability and possible triggering of immune response [70].

Most recently, novel and extremely powerful genetic tool-CRISPR (clustered regularly interspaced short palindromic repeat) - Cas9 (CRISPR-associated nuclease 9) system, adopted from bacteria defense mechanism against viral infection, has opened new possibilities to specifically knock-out any desired gene at the genomic level. In brief, the Cas9 nuclease is guided to specific genomic locus by single guide RNA sequence (sgRNA) where it induces double strand breaks (DSB) subsequently repaired by DNA repair mechanisms, such as non-homologous end joining DNA repair pathway (NHEJ). As the repair mechanism is an error-prone, insertions and deletions occur, what, in consequence, may lead to gene knock-out through changes in
mRNA reading frame and the introduction of the premature stop codon. CRISPR/Cas9 system, due to its specificity, efficiency, and simplicity, starts being a method of choice to study gene functions, also in tumor biology, where its therapeutic application was suggested (reviewed in: [71]). In our recent studies done on a model cell line, 293T, we confirmed the high effectiveness of CRISPR/Cas9 to specifically and efficiently target HO-1 (unpublished data). Although there are commercially available tools to generate HO-1 knock-out, there is a lack of studies evaluating the role of HO-1 in cancer using CRISPR/Cas9 technology, what, most probably, is just a matter of time. Of importance, except undeniable advantages of CRISPR/Cas9 technology, its drawbacks, namely possible off-target effects, delivery methods and others (reviewed in: [72]), have to be also taken into account when considering the experimental or therapeutic application of CRISPR/Cas9 system.

\section{Pharmacological inhibition of HO-1 activity}

Another approach is based on the inhibition of $\mathrm{HO}$ activity using pharmacological agents, namely: well-known and characterized metalloporphyrins like zinc protoporphyrin (ZnPPIX), tin protoporphyrin (SnPPIX) or chromium protoporphyrin (CrPPIX) and imidazole-based compounds (Fig. 3).

Metalloporphyrins (MPs) are heme analogues composed of protoporphyrin IX in which iron atom is exchanged by another component. The structure of these molecules

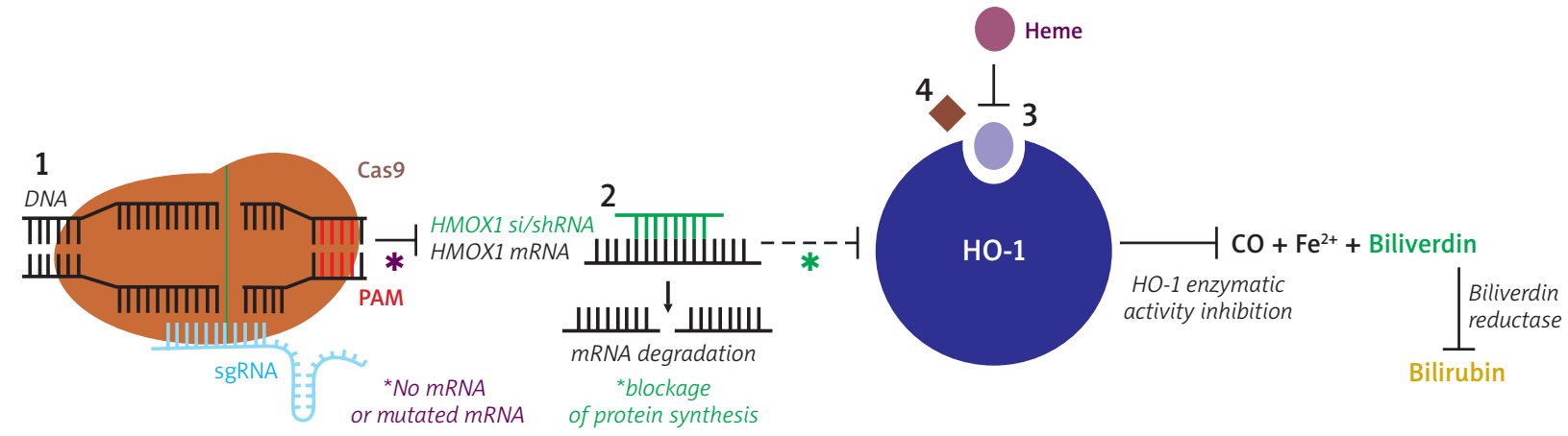

GENETIC INHIBITION

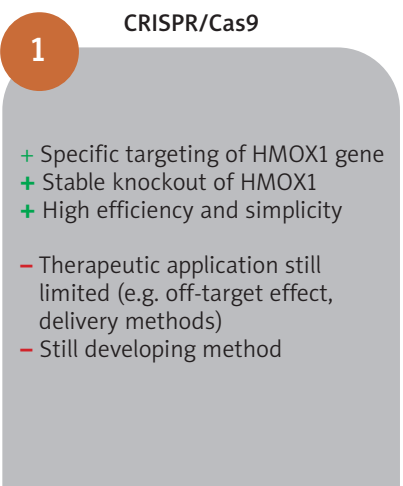

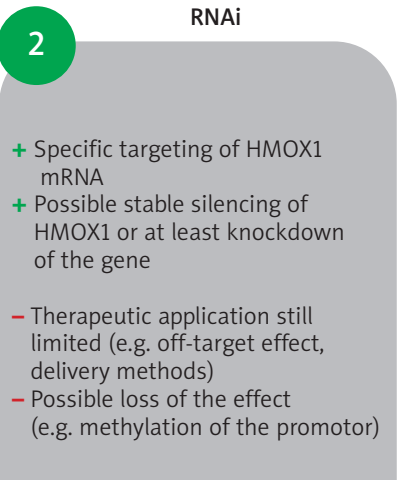
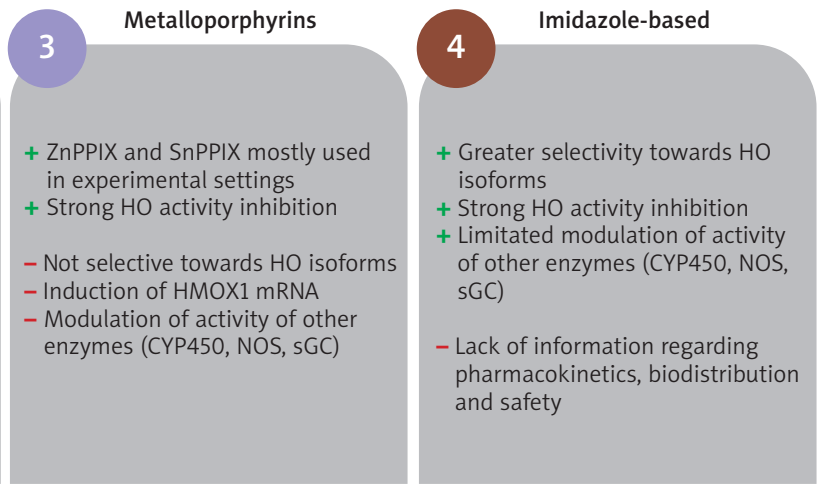

Fig. 3. Tools for HO-1 inhibition. Both genetic and pharmacological tools for HO-1 inhibition are available; however, all exert some disadvantages. Still more basic studies are necessary to identify the new, "ideal" specific inhibitors of HO-1 activity and/or to broaden our knowledge about CRISPR/Cas9 technology or siRNA targeting HO-1 
allows them to bind in the heme binding pocket of $\mathrm{HO}$ and instead of activation of the enzyme, they competitively inhibit its activity [73]. MPs have a much higher binding affinity than heme to $\mathrm{HO}$ isoforms and are not oxidatively degraded as they have no oxygen-binding capacity [74]. Majority of MPs have been shown to inhibit $\mathrm{HO}$ activity in vitro and in vivo, but protoporphyrins with nickel (Ni), copper (Cu), and magnesium (Mg) as central atoms do not affect $\mathrm{HO}$ activity. Oppositely, CoPPIX acts as a stimulant of $\mathrm{HO}$ activity and it is strong inducer of $\mathrm{HO}-1$ expression This effect results from the robust induction of $\mathrm{HO}-1$ gene expression through the repression of Bach1 and upregulation of the Nrf2 protein [75]. Another study showed also the involvement of FOXO1 transcription factor in COPPIX-mediated regulation of HO-1 expression [76].

Numerous experimental studies confirmed the usefulness of MPs in decreasing $\mathrm{HO}$ activity. For instance, treatment of cultured melanoma tumor with SnPPIX markedly enhanced the efficiency of photodynamic therapy [77] and attenuated Kaposi sarcoma growth in vivo [78]. Moreover, ZnPPIX significantly increased photodynamic therapy-mediated cytotoxicity towards colon carcinoma and ovarian carcinoma cells [12]. Inhibition of HO-1 by ZnPPIX increased the sensitivity of nasopharyngeal carcinoma cells to radiotherapy [79] and intensified effectiveness of cisplatin in liver cancer both in vitro and in vivo [80].

Unfortunately, due to the long list of disadvantages (Fig. 3, Table 1) the experimental and medical application of MPs is strongly limited. One of the major drawback is the photoreactivity of most of the MPs which may cause phototoxicity leading to tissue and even organ damage [74, 81]. Moreover, they are highly lipophilic, thus insoluble in aqueous solutions. It was partially overcome by

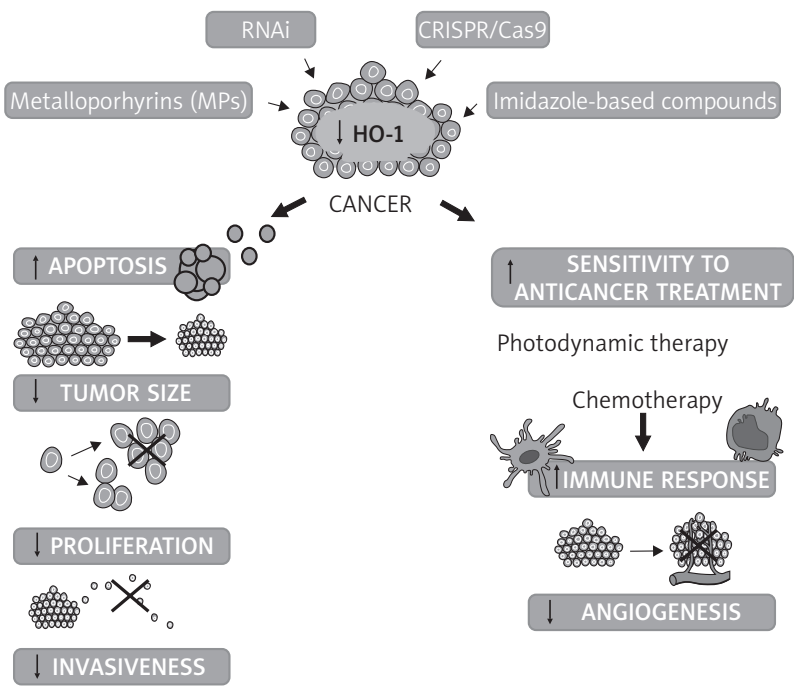

Fig. 4. The effect of HO-1 inhibition on tumor development and sensitivity to anticancer therapies. In cancer cells, the inhibition of HO-1 using metalloporphyrins (MPs), imidazole-based compounds, RNAi or CRISPR/Cas9 system can affect tumor development in various manner, both directly and indirectly. Directly, it may lead to increased apoptosis, impaired proliferation and invasiveness and reduction of the tumor size. The indirect effect is associated with increased sensitivity to anticancer treatments, like photo-, chemo- and radio- therapy by stimulation of the immune system and enhanced angiogenesis conjugation of the polyethylene-glycol [82] or amphiphilic styrene-maleic acid copolymer [83] to ZnPPIX, which resulted in the creation of water-soluble agents with potent $\mathrm{HO}$ inhibitory effect (PEG-ZnPPIX and SMA-ZnPPIX, respectively). Importantly, the anti-tumor effectiveness of these compounds was confirmed both in vitro and in vivo, without any apparent side effects [84, 85]. Nevertheless, several disadvantages are hard to overcome by any modification. One of them is a lack of selectivity. MPs not only inhibit the activity of both isoenzymes of $\mathrm{HO}[86]$ but because of the similarity of structure to heme, most of them also affect other heme-dependent enzymes like nitric oxide synthases (NOS), soluble guanylyl cyclase (sGC) or cytochromes P450 (CYPs) [87, 88]. Paradoxically, they also induce HO-1 on mRNA and protein level [9, 89, 90, 91]. These wide-ranging actions cause potential concerns in the interpretation of effects ascribed to MPs. Moreover, many undesired, independent of $\mathrm{HO}$ inhibition events were reported as the results of ZnPPIX and SnPPIX action. Interestingly, Wang et al. observed that cytotoxic effect of ZnPPIX on human ovarian and prostate cancer cell lines was altered neither by overexpression nor by knockout of HO-1, suggesting HO-1 independent effect of this inhibitor [92]. Similarly, in human colon [93], hepatoma and chronic myelogenous leukemia cell lines, as well as in BCL1 (B-cell leukemia/lymphoma 1)-tumor bearing mice [94], decrease in cancer cells proliferation and growth of tumors in vivo after ZnPPIX treatment was a direct consequence of cyclin D1 suppression and independent of HO-1 inhibition.

It has to be emphasized that $\mathrm{HO}$ inhibitors may act not only on tumor cells directly, but may also influence other cell types, including inflammatory cells (Fig. 4). Arnold et al. [14] have investigated the role of HO-1 expressing FAP+/ CD45+ macrophages associated with immunosuppression in tumorigenesis. Importantly they showed, that inhibition of HO-1 by Sn mesoporphyrin (SnMP) caused the immune-dependent arrest of tumor growth that was similar to the FAP+/CD45+ cells depletion.

Also $T$ cell can play a role in immune suppression. A few years ago it was shown not only that $\mathrm{HO}-1$ is highly expressed in a specific subpopulation of Treg cells but also that this subpopulation is accumulating in the glioma tumor during the progression of the disease and most likely play an immunosuppressive role [95]. Moreover, another group showed that overexpression of HO-1 in Treg cells is associated with better survival in hypoxia, typical for tumors, and inhibition of HO-1 using SnPP is able to revoke this effect, suggesting a protective role of the enzyme. Importantly, in in vivo experiment, SnPP treatment reduced the number of Treg cells in brain tumor microenvironment, what increased survival of the tumor-bearing mice [96].

Interestingly, systemic application of ZnPPIX in vivo into neuroblastoma-bearing mice as well as injection of neuroblastoma cell line NXS2 pre-treated with ZnPPIX into syngeneic mouse model significantly improved CD4+ and CD8+ T-effector cells functions and maturation of dendritic cells (DCs) via increased production of IL-10 [97]. In a mouse model of breast cancer, inhibition of HO-1 by ZnPPIX was associated with polarization of tumor-associated macrophages (TAM) towards M1-like phenotype (CD11b+ 
Table 1. Comparison of $\mathrm{HO}$ inhibitors

\begin{tabular}{|c|c|c|}
\hline & Metalloporphyrins & Imidazole-based compounds \\
\hline \multirow[t]{4}{*}{ Examples } & - Protoporphyrins: ZnPP, SnPP, CrPP & - QC-1 (azalanstat) \\
\hline & - Mesoporphyrins: ZnMP, SnMP, CrMP & - QC-13 \\
\hline & - Deuteroporphyrins: ZnDP, SnDP, CrDP & - QC-308 \\
\hline & - Modifications: PEG-ZnPP, SMA-ZnPP & \\
\hline \multirow[t]{5}{*}{ Major features } & - Competitive inhibitors & - Non-competitive inhibitors \\
\hline & - "Classical" protoporphyrins are water insoluble, & - Water soluble \\
\hline & $\begin{array}{l}\text { - "Modified" protoporphyrins: PEG-ZnPP and SMA-ZnPP } \\
\text { are water soluble and are claimed to be more available }\end{array}$ & - Some exert selectivity against $\mathrm{HO}$ isoforms \\
\hline & - Potent inhibitors of $\mathrm{HO}$ activity & \\
\hline & - Well-studied & \\
\hline \multirow[t]{6}{*}{ Adverse effects } & - Not selective towards $\mathrm{HO}$ isoforms & - Not studied widely in vitro and in vivo \\
\hline & - Inducers of HO-1 expression & - Not tested in clinical studies \\
\hline & - Modulators of NOS, sGC, and CYPS & \\
\hline & - Photosensitizers & \\
\hline & - Limited bioavailability & \\
\hline & - Inhibitors of IDO, cyclin D1 and WNT/ $\beta$-catenin (ZnPP) & \\
\hline
\end{tabular}

F4/80low (D206-) with anti-tumorigenic effectiveness [98]. Interestingly, recent study done by the group of Di Biase et al. showed that fasting-mimicking diet combined with chemotherapy triggers immune response against breast and melanoma cancer via an increase in lymphoid progenitor cells and CD8+ cytotoxic T lymphocytes. This, at least partially, was associated with downregulation of HO-1, also confirmed by the application of ZnPPIX [99].

Although all described so far effects of metalloporphyrins on anti-tumor immune response have been ascribed to specific HO-1 inhibition, one cannot exclude their unspecific mode of action. Indeed, Metz et al. have demonstrated that ZnPPIX specifically inhibits indoleamine 2,3-dioxygenase (IDO) enzyme, which is thought to favor immune escape in many types of tumors. The application of ZnPPIX and, as the results, inhibition of IDO in vivo in melanoma-bearing mice, resulted in anti-tumor effectiveness related to T-cell function, rather than HO-1 inhibition [100].

In order to enhance the progress in understanding the role of HO-1 in different pathological conditions, new, non-porphyrin-based class of $\mathrm{HO}$ inhibitors, without some drawbacks of MPs, were synthesized and evaluated. First was azalanstat which is an imidazole-dioxolane compound [101]. Based on its structure, and by changing the substituents, many new, preferentially more selective for $\mathrm{HO}-1$ isoform inhibitors were designed [102, 103]. Noteworthy, some of the compounds exhibit low or even no inhibitory activity on NOS, CYPS, and SGC, what is one of the major advantages over MPs [104] (Fig. 3, Table 1).

For the last couple of years, a lot of new inhibitors were developed, starting with QC-308 [105] or QC-13 [106]. All of these inhibitors differ in their potency to inhibit $\mathrm{HO}$ activity, their stability, eventual cytotoxicity and specificity [88]. One of the things that combine them all is the proposed mechanism of action. Imidazole-dioxolane derivatives act as non-competitive agents by binding to the distal side of heme and eventually causing displacement of the critical for catalysis (heme oxidation) water ligand [107]. Moreover, developed inhibitors are soluble and stable in physiological solutions [88]. Potent antitumor effect of imidazole-based HO-1 inhibitors was confirmed recently in prostate and breast cancer cell lines, however, the concentration of used compounds was quite high [108]. Additionally, promising antitumor effects were obtained for compound referred as OB-24. Inhibition of HO-1 activity in hormone-refractory prostate cancer resulted in decreased proliferation, viability and invasiveness of cancer cell in vitro. Furthermore, treatment of tumor-bearing mice with OB-24 significantly reduced growth and metastasis of tumors in vivo and this effect was potentiated together with the treatment with Taxol [109].

Nevertheless, we still lack detailed data on the pharmacokinetics, biodistribution and safety of this new class of inhibitors. For the last few years, our group was also involved in the study concerning searching for the new $\mathrm{HO}$ activity inhibitors. Until this day, we obtained some promising results which still require further validation.

\section{Summary}

As summarized in this review, inhibition of HO-1 can be considered as a potential anticancer strategy in various cancer types through several possible mechanisms (Fig. 4). Genetic inhibition of HMOX1 by shRNA approach, although has shown promising, anticancer effectiveness is still far from the therapeutic application. On the other hand, pharmacological inhibition of $\mathrm{HO}$ activity was shown to be effective, but due to the several limitations of known metalloporphyrin inhibitors, the advantage of this strate- 
gy is also doubtful. A better alternative may provide novel, small inhibitors of $\mathrm{HO}$ activity; however, a substantial number of studies is still needed to drive any conclusions about their anticancer effectiveness and potential therapeutic application.

This work was supported by the grant from the National Centre for Research and Development (PBS2I $B 7 / 15 / 2014)$ and from the National Science Centre (2016/21/B/NZ1/00293). Faculty of Biochemistry, Biophysics and Biotechnology of Jagiellonian University is a partner of the Leading National Research Center (KNOW) supported by the Ministry of Science and Higher Education. Figures were produced using Servier Medical Art (http:// www.servier.com).

\section{References}

1. Ponka P. Cell biology of heme. Am J Med Sci 1999; 318: 241-56.

2. Kumar S, Bandyopadhyay U. Free heme toxicity and its detoxification systems in human. Toxicol Lett 2005; 157: 175-88.

3. Tenhunen R, Marver HS, Schmid R. The enzymatic conversion of heme to bilirubin by microsomal heme oxygenase. Proc Natl Acad Sci USA 1968; 61: 748-55.

4. Hayashi S, Omata Y, Sakamoto H, Higashimoto Y, Hara T, Sagara Y, Noguchi M. Characterization of rat heme oxygenase-3 gene. Implication of processed pseudogenes derived from heme oxygenase-2 gene. Gene 2004; 336: 241-50.

5. Loboda A, Damulewicz M, Pyza E, Jozkowicz A, Dulak J. Role of Nrf2/HO-1 system in development, oxidative stress response and diseases: an evolutionarily conserved mechanism. Cell Mol Life Sci 2016; 73: 3221-47.

6. Loboda A, Jazwa A, Grochot-Przeczek A, Rutkowski AJ, Cisowski J, Agarwal A, Jozkowicz A, Dulak J. Heme oxygenase-1 and the vascular bed: from molecular mechanisms to therapeutic opportunities. Antioxid Redox Signal 2008; 10: 1767-812.

7. Deshane J, Chen S, Caballero S, et al. Stromal cell-derived factor 1 promotes angiogenesis via a heme oxygenase 1-dependent mechanism. J Exp Med 2007; 204: 605-18.

8. Dulak J, Jozkowicz A, Foresti R, et al. Heme oxygenase activity modulates vascular endothelial growth factor synthesis in vascular smooth muscle cells. Antioxid Redox Signal 2002; 4: 22940.

9. Loboda A, Jozkowicz A, Dulak J. HO-1/CO system in tumor growth, angiogenesis and metabolism - Targeting HO-1 as an anti-tumor therapy. Vascul Pharmacol 2015; 74: 11-22.

10. Berberat PO, Dambrauskas Z, Gulbinas A, et al. Inhibition of heme oxygenase-1 increases responsiveness of pancreatic cancer cells to anticancer treatment. Clin Cancer Res 2005; 11: 3790-8.

11. Kongpetch S, Kukongviriyapan V, Prawan A, Senggunprai L, Kukongviriyapan U, Buranrat B. Crucial role of heme oxygenase-1 on the sensitivity of cholangiocarcinoma cells to chemotherapeutic agents. PLoS One 2012; 7: e34994.

12. Nowis D, Legat M, Grzela T, et al. Heme oxygenase-1 protects tumor cells against photodynamic therapy-mediated cytotoxicity. Oncogene 2006; 25: 3365-74.

13. Nishie A, Ono M, Shono T, et al. Macrophage infiltration and heme oxygenase-1 expression correlate with angiogenesis in human gliomas. Clin Cancer Res 1999; 5: 1107-13.

14. Arnold JN, Magiera L, Kraman M, Fearon DT. Tumoral immune suppression by macrophages expressing fibroblast activation protein-alpha and heme oxygenase-1. Cancer Immunol Res 2014; 2: 121-6.

15. Andersen MH, Sorensen RB, Brimnes MK, Svane IM, Becker JC, thor Straten P. Identification of heme oxygenase-1-specific regulatory CD8+ T cells in cancer patients. J Clin Invest 2009; 119: 2245-56.
16. Torisu-Itakura H, Furue M, Kuwano M, Ono M. Co-expression of thymidine phosphorylase and heme oxygenase-1 in macrophages in human malignant vertical growth melanomas. Jpn J Cancer Res 2000; 91: 906-10.

17. Ryter SW, Alam J, Choi AM. Heme oxygenase-1/carbon monoxide: from basic science to therapeutic applications. Physiol Rev 2006; 86: 583-650.

18. Lin Q, Weis S, Yang G, et al. Heme oxygenase-1 protein localizes to the nucleus and activates transcription factors important in oxidative stress. J Biol Chem 2007; 282: 20621-33.

19. Hsu FF, Yeh CT, Sun YJ, Chiang MT, Lan WM, Li FA, Lee WH, Chau LY. Signal peptide peptidase-mediated nuclear localization of heme oxygenase-1 promotes cancer cell proliferation and invasion independent of its enzymatic activity. Oncogene 2015; 34: 2410-1.

20. Sacca P, Meiss R, Casas G, Mazza O, Calvo JC, Navone N, Vazquez E. Nuclear translocation of haeme oxygenase-1 is associated to prostate cancer. Br J Cancer 2007; 97: 1683-9.

21. Gandini NA, Fermento ME, Salomon DG, et al. Nuclear localization of heme oxygenase-1 is associated with tumor progression of head and neck squamous cell carcinomas. Exp Mol Pathol 2012; 93: 237-45.

22. Sunamura M, Duda DG, Ghattas MH, et al. Heme oxygenase-1 accelerates tumor angiogenesis of human pancreatic cancer. Angiogenesis 2003; 6: 15-24.

23. Was H, Cichon T, Smolarczyk R, et al. Overexpression of heme oxygenase-1 in murine melanoma: increased proliferation and viability of tumor cells, decreased survival of mice. Am J Pathol 2006; 169: 2181-98.

24. Li MY, Yip J, Hsin MK, Mok TS, Wu Y, Underwood MJ, Chen GG. Haem oxygenase-1 plays a central role in NNK-mediated lung carcinogenesis. Eur Respir J 2008; 32: 911-23.

25. Hill M, Pereira V, Chauveau C, et al. Heme oxygenase-1 inhibits rat and human breast cancer cell proliferation: mutual cross inhibition with indoleamine 2,3-dioxygenase. FASEB J 2005; 19: 1957 68.

26. Gueron G, De Siervi A, Ferrando M, et al. Critical role of endogenous heme oxygenase 1 as a tuner of the invasive potential of prostate cancer cells. Mol Cancer Res, 2009; 7: 1745-55.

27. Na HK, Surh YJ. Oncogenic potential of Nrf2 and its principal target protein heme oxygenase-1. Free Radic Biol Med, 2014; 67: 353-65.

28. Liu ZM, Chen GG, Ng EK, Leung WK, Sung JJ, Chung SC. Upregulation of heme oxygenase-1 and p21 confers resistance to apoptosis in human gastric cancer cells. Oncogene 2004; 23: 503-13.

29. Busserolles J, Megias J, Terencio MC,Alcaraz MJ. Heme oxygenase-1 inhibits apoptosis in Caco-2 cells via activation of Akt pathway. Int J Biochem Cell Biol 2006; 38: 1510-7.

30. Zhu XF, Li W, Ma JY, et al. Knockdown of heme oxygenase-1 promotes apoptosis and autophagy and enhances the cytotoxicity of doxorubicin in breast cancer cells. Oncol Lett 2015; 10: 2974 80.

31. Andreadi CK, Howells LM, Atherfold PA, Manson MM. Involvement of Nrf2, p38, B-Raf, and nuclear factor-kappaB, but not phosphatidylinositol 3-kinase, in induction of hemeoxygenase-1 by dietary polyphenols. Mol Pharmacol 2006; 69: 1033-40.

32. White E. The role for autophagy in cancer. J Clin Invest 2015; 125: 42-6.

33. Banerjee P, Basu A, Wegiel B, et al. Heme oxygenase-1 promotes survival of renal cancer cells through modulation of apoptosisand autophagy-regulating molecules. J Biol Chem 2012; 287: 32113-23.

34. Tan Q, Wang H, Hu Y, et al. Src/STAT3-dependent heme oxygenase-1 induction mediates chemoresistance of breast cancer cells to doxorubicin by promoting autophagy. Cancer Sci 2015; 106: 1023-32.

35. Loboda A, Jazwa A, Wegiel B, Jozkowicz A, Dulak J. Heme oxygenase-1-dependent and -independent regulation of angiogenic genes expression: effect of cobalt protoporphyrin and cobalt chloride on VEGF and IL-8 synthesis in human microvascular endothelial cells. Cell Mol Biol (Noisy-le-grand) 2005; 51: 347-55.

36. Mcallister SC, Hansen SG, Ruhl RA, Raggo CM, DeFilippis VR, Greenspan D, Früh K, Moses AV. Kaposi sarcoma-associated her- 
pesvirus (KSHV) induces heme oxygenase-1 expression and activity in KSHV-infected endothelial cells. Blood 2004; 103: $3465-$ 73.

37. Miyake M, Fujimoto K, Anai S, et al. Heme oxygenase-1 promotes angiogenesis in urothelial carcinoma of the urinary bladder. Oncol Rep 2011; 25: 653-60.

38. Birrane G, Li H, Yang S, Tachado SD, Seng S. Cigarette smoke in duces nuclear translocation of heme oxygenase 1 (HO-1) in prostate cancer cells: nuclear HO-1 promotes vascular endothelial growth factor secretion. Int J Oncol 2013; 42: 1919-28.

39. Ciesla M, Marona P, Kozakowska M, et al. Heme Oxygenase-1 Controls an HDAC4-miR-206 Pathway of Oxidative Stress in Rhabdomyosarcoma. Cancer Res 2016; 76: 5707-18.

40. Hirai K, Sasahira T, Ohmori H, Fujii K, Kuniyasu H. Inhibition of heme oxygenase-1 by zinc protoporphyrin IX reduces tumor growth of LL/2 lung cancer in C57BL mice. Int J Cancer 2007; 120: 500-5.

41. Lee SS, Yang SF, Tsai CH, Chou MC, Chou MY, Chang YC. Upregulation of heme oxygenase-1 expression in areca-quid-chewing-associated oral squamous cell carcinoma. J Formos Med Assoc 2008; 107: 355-63.

42. Tsai JR, Wang HM, Liu PL, et al. High expression of heme oxygenase- 1 is associated with tumor invasiveness and poor clinical outcome in non-small cell lung cancer patients. Cell Oncol (Dordr) 2012; 35: 461-71

43. Skrzypek K, Tertil M, Golda S, et al. Interplay between heme ox ygenase-1 and miR-378 affects non-small cell lung carcinoma growth, vascularization, and metastasis. Antioxid Redox Signal 2013; 19: 644-60.

44. Kozakowska M, Ciesla M, Stefanska A, et al. Heme oxygenase-1 inhibits myoblast differentiation by targeting myomirs. Antioxid Redox Signal 2012; 16: 113-27.

45. Tomlinson IP, Alam NA, Rowan AJ, et al. Germline mutations in FH predispose to dominantly inherited uterine fibroids, skin leiomyomata and papillary renal cell cancer. Nat Genet 2002; 30: 406 10.

46. Yang M, Soga T, Pollard PJ, Adam J. The emerging role of fumarate as an oncometabolite. Front Oncol 2012; 2: 85.

47. Adam J, Hatipoglu E, O'Flaherty L, et al. Renal cyst formation in Fh1-deficient mice is independent of the Hif/Phd pathway: roles for fumarate in KEAP1 succination and Nrf2 signaling. Cancer Cell 2011; 20: 524-37

48. Frezza C, Zheng L, Folger O, et al. Haem oxygenase is synthetically lethal with the tumour suppressor fumarate hydratase. Nature 2011; 477: 225-8.

49. Nijman SM. Synthetic lethality: general principles, utility and detec tion using genetic screens in human cells. FEBS Lett 2011; 585: 1-6.

50. Exner M, Minar E, Wagner O, Schillinger M. The role of heme oxygenase-1 promoter polymorphisms in human disease. Free Radic Biol Med 2004; 37: 1097-104.

51. Taha H, Skrzypek K, Guevara I, et al. Role of heme oxygenase-1 in human endothelial cells: lesson from the promoter allelic variants. Arterioscler Thromb Vasc Biol 2010; 30: 1634-41.

52. Vashist YK, Uzungolu G, Kutup A, et al. Heme oxygenase-1 germ line GTn promoter polymorphism is an independent prognosticator of tumor recurrence and survival in pancreatic cancer. J Surg Oncol 2011; 104: 305-11.

53. Sawa T, Mounawar M, Tatemichi M, Gilibert I, Katoh T, Ohshima $\mathrm{H}$. Increased risk of gastric cancer in Japanese subjects is associated with microsatellite polymorphisms in the heme oxygenase-1 and the inducible nitric oxide synthase gene promoters. Cancer Lett 2008; 269: 78-84.

54. Hong CC, Ambrosone CB, Ahn J, et al. Genetic variability in iron-related oxidative stress pathways (Nrf2, NQ01, NOS3, and $\mathrm{HO}-1$ ), iron intake, and risk of postmenopausal breast cancer. Cancer Epidemiol Biomarkers Prev 2007; 16: 1784-94.

55. Hu JL, Li ZY, Liu W, Zhang RG, Li GL, Wang T, Ren JH, Wu G. Poly morphism in heme oxygenase-1 (HO-1) promoter and alcohol are related to the risk of esophageal squamous cell carcinoma on Chinese males. Neoplasma 2010; 57: 86-92.

56. Tang D, Tang WJ, Shi XL, Li WP, Zhou H, Lu LM, Tao L. Association of the microsatellite (GT)n repeat polymorphisms of the HO-1 gene promoter and corresponding serum levels with the risk of laryngeal squamous cell carcinoma. Acta Otolaryngol 2016; 136: 806-11.

57. Kikuchi A, Yamaya M, Suzuki S, et al. Association of susceptibility to the development of lung adenocarcinoma with the heme oxygenase-1 gene promoter polymorphism. Hum Genet 2005; 116: 354-60.

58. Jiraskova A, Novotny J, Novotny L, et al. Association of serum bilirubin and promoter variations in HMOX1 and UGT1A1 genes with sporadic colorectal cancer. Int J Cancer 2012; 131: 1549-55.

59. Zhang L, Song FF, Huang YB, et al. Association between the (GT)n polymorphism of the HO-1 gene promoter region and cancer risk: a meta-analysis. Asian Pac J Cancer Prev 2014; 15: 4617-22.

60. Abdelrahim M, Safe S, Baker C, et al. RNAi and cancer: Implications and applications. J RNAi Gene Silencing, 2006; 2: 136-45.

61. Kim HR, Kim S, Kim EJ, et al. Suppression of Nrf2-driven heme oxygenase-1 enhances the chemosensitivity of lung cancer A549 cells toward cisplatin. Lung Cancer 2008; 60: 47-56.

62. Mayerhofer M, Gleixner KV, Mayerhofer J, et al. Targeting of heat shock protein 32 (Hsp32)/heme oxygenase-1 (HO-1) in leukemic cells in chronic myeloid leukemia: a novel approach to overcome resistance against imatinib. Blood 2008; 111: 2200-10.

63. Rushworth SA, Macewan DJ. HO-1 underlies resistance of AML cells to TNF-induced apoptosis. Blood 2008; 111: 3793-801.

64. Wei S, Wang Y, Chai Q, Fang Q, Zhang Y, Wang J. Potential crosstalk of Ca2+-ROS-dependent mechanism involved in apoptosis of Kasumi-1 cells mediated by heme oxygenase-1 small interfering RNA. Int J Oncol 2014; 45: 2373-84.

65. Ren QG, Yang SL, Hu JL, Li PD, Chen YS, Wang QS. Evaluation of HO-1 expression, cellular ROS production, cellular proliferation and cellular apoptosis in human esophageal squamous cell carcinoma tumors and cell lines. Oncol Rep 2016; 35: 2270-6.

66. Zhe N, Wang J, Chen S, Lin X, Chai Q, Zhang Y, Zhao J, Fang Q. Heme oxygenase-1 plays a crucial role in chemoresistance in acute myeloid leukemia. Hematology 2015; 20: 384-91.

67. Paez AV, Pallavicini C, Schuster F, et al. Heme oxygenase-1 in the forefront of a multi-molecular network that governs cell-cell contacts and filopodia-induced zippering in prostate cancer. Cell Death Dis 2016; 7: e2570.

68. Sass G, Leukel P, Schmitz V, et al. Inhibition of heme oxygenase 1 expression by small interfering RNA decreases orthotopic tumor growth in livers of mice. Int J Cancer 2008; 123: 1269-77.

69. Kang KA, Piao MJ, Kim KC, et al. Epigenetic modification of Nrf2 in 5-fluorouracil-resistant colon cancer cells: involvement of TET-dependent DNA demethylation. Cell Death Dis 2014; 5: e1183.

70. Aagaard L, Rossi JJ. RNAi therapeutics: principles, prospects and challenges. Adv Drug Deliv Rev 2007; 59: 75-86.

71. Yi L,Li J, CRISPR-Cas9 therapeutics in cancer: promising strategies and present challenges. Biochim Biophys Acta 2016; 1866: 197207.

72. Peng R, Lin G,Li J, Potential pitfalls of CRISPR/Cas9-mediated genome editing. FEBS J 2016; 283: 1218-31.

73. Maines MD. Zinc protoporphyrin is a selective inhibitor of heme oxygenase activity in the neonatal rat. Biochim Biophys Acta 1981; 673: 339-50.

74. Schulz S, Wong RJ, Vreman HJ, Stevenson DK. Metalloporphyrins - an update. Front Pharmacol 2012; 3: 68.

75. Shan Y, Lambrecht RW, Donohue SE, Bonkovsky HL. Role of Bach1 and Nrf2 in up-regulation of the heme oxygenase-1 gene by cobalt protoporphyrin. FASEB J 2006; 20: 2651-3.

76. Liu X, Cui Y, Li M, Xu H, Zuo J, Fang F, Chang Y. Cobalt protoporphyrin induces HO-1 expression mediated partially by FOXO1 and reduces mitochondria-derived reactive oxygen species production. PLoS One 2013; 8: e80521.

77. Frank J, Lornejad-Schäfer MR, Schöffl H, Flaccus A, Lambert C, Biesalski HK. Inhibition of heme oxygenase-1 increases responsiveness of melanoma cells to ALA-based photodynamic therapy. Int J Oncol 2007; 31: 1539-45.

78. Marinissen MJ, Tanos T, Bolós M, de Sagarra MR, Coso OA, Cuadrado A. Inhibition of heme oxygenase-1 interferes with the transforming activity of the Kaposi sarcoma herpesvirus-encoded G protein-coupled receptor. J Biol Chem 2006; 281: 11332-46. 
79. Shi L, Fang J. Implication of heme oxygenase-1 in the sensitivity of nasopharyngeal carcinomas to radiotherapy. J Exp Clin Cancer Res 2008; 27: 13.

80. Liu YS, Li HS, Qi DF, Zhang J, Jiang XC, Shi K, Zhang XJ, Zhang XH. Zinc protoporphyrin IX enhances chemotherapeutic response of hepatoma cells to cisplatin. World J Gastroenterol 2014; 20: 857282.

81. Vreman HJ, Ekstrand BC, Stevenson DK. Selection of metalloporphyrin heme oxygenase inhibitors based on potency and photo reactivity. Pediatr Res 1993; 33: 195-200.

82. Sahoo SK, Sawa T, Fang J, Tanaka S, Miyamoto Y, Akaike T, Maeda H. Pegylated zinc protoporphyrin: a water-soluble heme oxy genase inhibitor with tumor-targeting capacity. Bioconjug Chem 2002; 13: 1031-8.

83. Iyer AK, Greish K, Seki T, Okazaki S, Fang J, Takeshita K, Maeda H. Polymeric micelles of zinc protoporphyrin for tumor targeted delivery based on EPR effect and singlet oxygen generation. J Drug Target 2007; 15: 496-506

84. Fang J, Sawa T, Akaike T, Akuta T, Sahoo SK, Khaled G, Hamada A, Maeda $\mathrm{H}$. In vivo antitumor activity of pegylated zinc protoporphyrin: targeted inhibition of heme oxygenase in solid tumor. Cancer Res 2003; 63: 3567-74.

85. Herrmann H, Kneidinger M, Cerny-Reiterer S, et al. The Hsp32 inhibitors SMA-ZnPP and PEG-ZnPP exert major growth-inhibitory effects on D34+/CD38+ and CD34+/CD38- AML progenitor cells. Curr Cancer Drug Targets 2012; 12: 51-63.

86. Wong RJ, Vreman HJ, Schulz S, Kalish FS, Pierce NW, Stevenson DK. In vitro inhibition of heme oxygenase isoenzymes by metalloporphyrins. J Perinatol 2011; 31 Suppl 1: S35-41.

87. Appleton SD, Chretien ML, Mclaughlin BE, Vreman HJ, Stevenson DK, Brien JF, Nakatsu K, Maurice DH, Marks GS. Selective inhibition of heme oxygenase, without inhibition of nitric oxide synthase or soluble guanylyl cyclase, by metalloporphyrins at low concentrations. Drug Metab Dispos 1999; 27: 1214-9.

88. Kinobe RT, Dercho RA, Nakatsu K. Inhibitors of the heme oxygenase - carbon monoxide system: on the doorstep of the clinic? Can J Physiol Pharmacol 2008; 86: 577-99.

89. Kwok SC. Zinc Protoporphyrin Upregulates Heme Oxygenase-1 in PC-3 Cells via the Stress Response Pathway. Int J Cell Biol 2013; 2013: 162094

90. Sardana MK, Kappas A. Dual control mechanism for heme oxygenase: tin(IV)-protoporphyrin potently inhibits enzyme activity while markedly increasing content of enzyme protein in liver. Proc Natl Acad Sci USA 1987; 84: 2464-8.

91. Yang G, Nguyen X, Ou J, Rekulapelli P, Stevenson DK, Dennery PA. Unique effects of zinc protoporphyrin on $\mathrm{HO}-1$ induction and apoptosis. Blood 2001; 97: 1306-13.

92. Wang S, Avery JE, Hannafon BN, Lind SE, Ding WQ. Zinc protoporphyrin suppresses cancer cell viability through a heme oxygenase-1-independent mechanism: the involvement of the Wnt/ beta-catenin signaling pathway. Biochem Pharmacol 2013; 85: 1611-8.

93. Nowis D, Bugajski M, Winiarska M, et al. Zinc protoporphyrin IX, a heme oxygenase-1 inhibitor, demonstrates potent antitumor effects but is unable to potentiate antitumor effects of chemotherapeutics in mice. BMC Cancer 2008; 8: 197.

94. La P, Fernando AP, Wang Z, Salahudeen A, Yang G, Lin Q, Wright CJ, Dennery PA. Zinc protoporphyrin regulates cyclin D1 expression independent of heme oxygenase inhibition. J Biol Chem 2009; 284: 36302-11.

95. El Andaloussi A, Lesniak MS. CD4+ CD25+ FoxP3+ T-cell infiltration and heme oxygenase-1 expression correlate with tumor grade in human gliomas. J Neurooncol 2007; 83: 145-52.

96. Chauveau C, Remy S, Royer PJ, et al. Heme oxygenase-1 expression inhibits dendritic cell maturation and proinflammatory function but conserves IL-10 expression. Blood 2005; 106: 1694-702.

97. Fest S, Soldati R, Christiansen NM, et al. Targeting of heme oxygenase-1 as a novel immune regulator of neuroblastoma. Int J Cancer 2016; 138: 2030-42.

98. Deng R, Wang SM, Yin T, et al. Inhibition of tumor growth and alteration of associated macrophage cell type by an $\mathrm{HO}-1$ inhibitor in breast carcinoma-bearing mice. Oncol Res 2013; 20: 473-82.
99. Di Biase S, Lee C, Brandhorst S, et al. Fasting-Mimicking Diet Reduces HO-1 to Promote T Cell-Mediated Tumor Cytotoxicity. Cancer Cell, 2016; 30: 136-46.

100. Metz R, Duhadaway JB, Rust S, Munn DH, Muller AJ, Mautino M, Prendergast GC. Zinc protoporphyrin IX stimulates tumor immunity by disrupting the immunosuppressive enzyme indoleamine 2,3-dioxygenase. Mol Cancer Ther, 2010; 9: 1864-71.

101. Vlahakis JZ, Kinobe RT, Bowers RJ, Brien JF, Nakatsu K, Szarek WA. Synthesis and evaluation of azalanstat analogues as heme oxygenase inhibitors. Bioorg Med Chem Lett 2005; 15: 1457-61.

102. Vlahakis JZ, Hum M, Rahman MN, Jia Z, Nakatsu K, Szarek WA. Synthesis and evaluation of imidazole-dioxolane compounds as selective heme oxygenase inhibitors: effect of substituents at the 4-position of the dioxolane ring. Bioorg Med Chem 2009; 17 : 2461-75.

103. Vlahakis JZ, Kinobe RT, Bowers RJ, Brien JF, Nakatsu K, Szarek WA. Imidazole-dioxolane compounds as isozyme-selective heme oxygenase inhibitors. J Med Chem, 2006; 49: 4437-41.

104. Kinobe RT, Vlahakis JZ, Vreman HJ, Stevenson DK, Brien JF, Szarek WA, Nakatsu K. Selectivity of imidazole-dioxolane compounds for in vitro inhibition of microsomal haem oxygenase isoforms. Br J Pharmacol 2006. 147: 307-15.

105. Rahman MN, Vlahakis JZ, Vukomanovic D, Lee W, Szarek WA, Nakatsu K, Jia Z. A novel, "double-clamp" binding mode for human heme oxygenase-1 inhibition. PLoS One 2012; 7: e29514.

106. Pittala V, Salerno L, Romeo G, Modica MN, Siracusa MA. A focus on heme oxygenase-1 (HO-1) inhibitors. Curr Med Chem 2013; 20: 3711-32.

107. Rahman MN, Vlahakis JZ, Roman G, Vukomanovic D, Szarek WA, Nakatsu K, Jia Z. Structural characterization of human heme oxygenase-1 in complex with azole-based inhibitors. J Inorg Biochem 2010; 104: 324-30.

108. Salerno L, Pittala V, Romeo G, et al. Novel imidazole derivatives as heme oxygenase-1 (HO-1) and heme oxygenase-2 (HO-2) inhibitors and their cytotoxic activity in human-derived cancer cell lines. Eur J Med Chem 2015; 96: 162-72.

109. Alaoui-Jamali MA, Bismar TA, Gupta A, et al. A novel experimental heme oxygenase-1-targeted therapy for hormone-refractory prostate cancer. Cancer Res 2009; 69: 8017-24

\section{Address for correspondence}

\section{Agnieszka Łoboda and Józef Dulak}

Department of Medical Biotechnology

Faculty of Biochemistry, Biophysics and Biotechnology,

Jagiellonian University

Gronostajowa 7

30-387 Krakow, Poland

e-mail: agnieszka.loboda@uj.edu.pl

e-mail: jozef.dulak@uj.edu.pl 